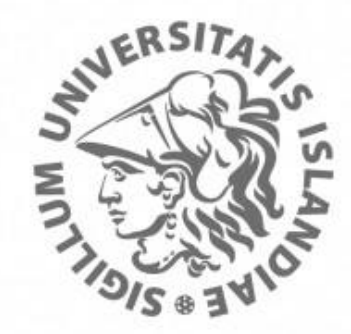

This is not the published version of the article / Petta er ekki útgefna útgáfa greinarinnar

Author(s)/Höf.: $\quad$ Sitek, A., Urbaneja Torres, M., Torfason, K., Gudmundsson, V., Bertoni, A., \& Manolescu, A.

Title/Titill: $\quad$ Excitons in core-shell nanowires with polygonal cross sections

Year/Útgáfuár: $\quad 2018$

Version/Útgáfa: $\quad$ Pre-print / Óritrýnt handrit

Please cite the original version:

Vinsamlega vísið til útgefnu greinarinnar:

Sitek, A., Urbaneja Torres, M., Torfason, K., Gudmundsson, V., Bertoni, A., \& Manolescu, A. (2018). Excitons in Core-Shell Nanowires with Polygonal Cross Sections. Nano Letters, 18(4), 2581-2589. doi:10.1021/acs.nanolett.8b00309

Rights/Réttur: $\quad$ Copyright @ 2018 American Chemical Society 


\title{
Excitons in core-shell nanowires with polygonal cross sections
}

\author{
Anna Sitek, ${ }^{1,2}$ Miguel Urbaneja Torres, ${ }^{1}$ Kristinn Torfason, ${ }^{1}$ \\ Vidar Gudmundsson, ${ }^{3}$ Andrea Bertoni, ${ }^{4}$ and Andrei Manolescu ${ }^{1}$ \\ ${ }^{1}$ School of Science and Engineering, Reykjavik University, \\ Menntavegur 1, IS-101 Reykjavik, Iceland \\ ${ }^{2}$ Department of Theoretical Physics, \\ Faculty of Fundamental Problems of Technology, \\ Wroclaw University of Science and Technology, \\ Wybrzėze Wyspiańskiego 27, 50-370 Wroclaw, Poland \\ ${ }^{3}$ Science Institute, University of Iceland, \\ Dunhaga 3, IS-107 Reykjavik, Iceland \\ ${ }^{4}$ Istituto Nanoscienze-CNR, Via Campi 213a, I-41125, Modena, Italy
}

\begin{abstract}
The distinctive prismatic geometry of semiconductor core-shell nanowires leads to complex localization patterns of carriers. Here, we describe the formation of optically active in-gap excitonic states induced by the interplay between localization of carriers in the corners and their mutual Coulomb interaction. To compute the energy spectra and configurations of excitons created in the conductive shell, we use a multi-electron numerical approach based on the exact solution of the multi-particle Hamiltonian for electrons in the valence and conduction bands, which includes the Coulomb interaction in a nonperturbative manner. We expose the formation of well separated quasidegenerate levels, and focus on the implications of the electron localization in the corners or on the sides of triangular, square and hexagonal cross sections. We obtain excitonic in-gap states associated with symmetrically distributed electrons in the spin singlet configuration. They acquire large contributions due to Coulomb interaction, and thus are shifted to much higher energies than other states corresponding to the conduction electron and the vacancy localized in the same corner. We compare the results of the multi-electron method with those of an electron-hole model and we show that the latter does not reproduce the singlet excitonic states. We also obtain the exciton lifetime and explain selection rules which govern the recombination process.
\end{abstract}




\section{INTRODUCTION}

The possibility of combining two different materials into one nanostructure allowed for on demand growth of systems with a huge variety of physical properties, some of which controllable to a high extent. An example of such structures are core-shell nanowires. They consist of a core, a wire itself, which is surrounded by a layer (shell) of a different material. The crystallographic structure usually imposes polygonal cross sections, most commonly hexagonal [1-3]. Still, other prismatic geometries, like square [4], triangular [5-10], or dodecagonal [11], have also been obtained more and more frequently.

In a thin polygonal ring, i.e., in the shell of a short core-shell wire, with $p$ sides and with sufficiently small asymmetries, the low-energy electrons are localized in the corners while the electrons in the following layer occupy the sides [12-15]. With spin degeneracy included, and in a single-particle description, each of these layers consists of $2 p$ spin-orbital states. Here we consider a polygonal ring with finite radial thickness, i.e., the thickness of the shell. An interesting aspect is that the energy separation between corner and side states increases with decreasing the shell thickness and/or the number of vertices whereas, at the same time, the energy dispersion of the corner and side states reduces. In particular, for triangular rings, the energy gap between corner and side states can become much larger than the room-temperature energy, while the corner states become nearly degenerate [16-19].

Consequently, in a core-shell nanowire with a thin prismatic shell the low-energy electrons form energy bands with respect to the longitudinal wavevector $k$, with localized transverse modes either along the prism edges or on the prism sides. Hence, such a structure can in fact host a bunch of parallel wires robust to many perturbations. In particular, prismatic core-shell nanowire can be interesting for obtaining multiple Majorana states at each of the two ends if the polygonal geometry is not damaged by external elements like gates, contacts, etc. [20].

The research on core-shell nanowires was triggered by the possibility they show for the optimization of solar cells [21-26] and antennas [27-29]. Other interesting applications are lasers [30-33] and light emitting diodes [6, 34, 35]. Although the optical properties of nanowires with polygonal cross sections have been widely investigated experimentally [3644], and theoretically [45-47], the attention has been mostly focused on hexagonal structures. Still, triangular core-multishell wires have also shown unique properties, such as a broad range of emitted radiation observed even at room temperature [48] and tunable to a high extent [6] which makes them perfect candidates for building blocks of multicolor light sources. Due to the emission in the infrared range $[8,10,49]$ they also show promise for optical communication. Moreover, core-shell triangular $\mathrm{p}-\mathrm{n}$ and $\mathrm{p}-\mathrm{i}-\mathrm{n}$ junctions show clear diode characteristics and their light absorption may be controlled through the variation of indium mole fraction in the intrinsic region [9].

In recent theoretical works some of us calculated optical absorption spectra of low-energy electrons confined in polygonal rings, considering independent electrons, and evaluating the implications of the polygon geometry on the transitions between corner and/or side states $[18,19]$. For electrons with mutual Coulomb interactions, and for appropriate material parameters, we showed the possibility of obtaining multi-electron states within the gap between corner and corner-side states [50]. These in-gap states are associated with pairs of electrons in a spin singlet configuration occupying the same corner area. The small energy dispersion of the corner states, spin selection rules, and the presence of the in-gap states allow for contactless control of the absorption by external electric or magnetic fields. 
In the present paper we study excitons confined in the shell of a prismatic nanowire, i.e., subjected to the tubular prismatic geometry which leads to the unique localization in the corners or on the sides of the cross sections. In principle, the confinement of the electrons and holes in the shell depends on the band alignment and on the effective potential at the interface with the core. Still, the band alignment may be controlled during the growth process through the appropriate combination of materials used, core diameter and side thickness. $[47,51,52]$ This allows for fabrication of core-shell wires in which electrons are accumulated only in the outer part [1] and form a conductive shell. Moreover, hollow nanowires, i.e., with vacuum inside, can be obtained by removing the core part with chemical methods $[2,3]$. We focus especially on such hollow systems and on the effects resulting from their polygonal cross sections. We treat the Coulomb interaction with a multi-electron method [50] with the inclusion of both valence and conduction bands. We consider electronic states with zero wavevector $k$. In particular, we show that for sufficiently narrow shells the excitonic energy spectrum consists of nearly degenerate levels corresponding to particular electron distributions. The two largest splittings within the spectrum result from the difference of Coulomb repulsion caused by the rearrangement of electrons between the corner areas and from the gap separating single-particle corner and side states in the conduction band. The latter one also splits excitonic states corresponding to the configurations when the excited electron is situated either in the corner or on the side of the polygon. In between these levels we obtain in-gap excitons, i.e., states associated with the conduction electron and the vacancy localized on the same corner which, due to the spin singlet configuration and then strong Coulomb repulsion, are shifted to much higher energies than the other states corresponding to equal particle distributions. Our main model includes only electrons within a configuration-interaction scheme with the Coulomb interaction treated by numerical diagonalization. For comparison, we also consider an effective electron-hole model, and show that this approach does not reproduce the in-gap excitons.

\section{THE MODEL}

We consider the transverse states of electrons confined in the shell of a core-shell nanowire. These are the states of a polygonal ring with a lateral thickness in the $(x, y)$ plane given by the width of the shell and with a short height in the growth direction, $z$, such that all electrons are in the lowest vertical (or longitudinal) mode. These are also the relevant states for the excitons created in an infinitely long nanowire at wavevector $k=0$ in a material with direct band gap.

The single-particle Hamiltonian consists of two elements, $H=H_{\mathrm{v}}+H_{\mathrm{c}}$, describing electrons in the valence and the conduction band, respectively. Assuming that the zero energy level corresponds to the top of the bulk valence band, each component takes the form

$$
H_{\mathrm{i}}=\frac{(-i \hbar \nabla+e \boldsymbol{A})^{2}}{2 m_{\mathrm{i}}^{*}}+\delta_{\mathrm{c}, \mathrm{i}} E_{\mathrm{b}}-g_{\mathrm{i}}^{*} \mu_{\mathrm{B}} \sigma_{z} B-e \boldsymbol{E} \cdot \boldsymbol{r},
$$

where the subscript $\mathrm{i}=\{\mathrm{v}, \mathrm{c}\}$ refers to one of the two bands, $m_{\mathrm{v}, \mathrm{c}}^{*}$ and $g_{\mathrm{v}, \mathrm{c}}^{*}$ are the effective masses of the electrons and the $\mathrm{g}$ factors in the valence and the conduction band, respectively, $E_{\mathrm{b}}$ is the (bulk) semiconductor band gap, $\mu_{\mathrm{B}}$ the Bohr magneton, $e$ the electron charge, and $\sigma_{z}$ stands for the $z$ Pauli matrix. $\boldsymbol{A}=B[-y, x, 0] / 2$ is the vector potential of an external 

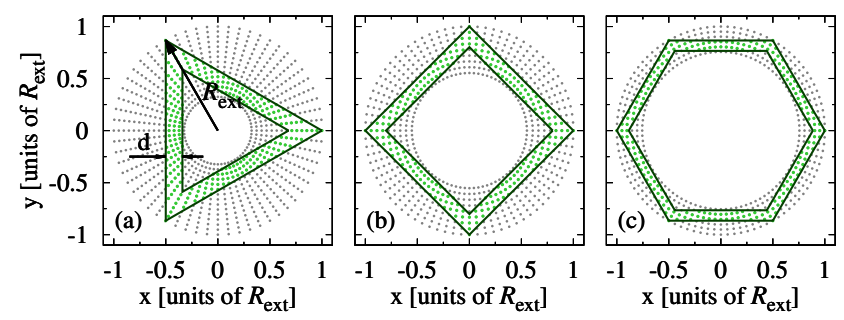

FIG. 1. Sample model - polygonal constraints applied on a polar grid. For visibility we reduced the number of site points.

uniform magnetic field $(B)$ in the longitudinal $z$ direction. We also consider a uniform electric field in the plane $(x, y)$, making an angle $\varphi$ with the $x$ axis, $\boldsymbol{E}=E[\cos (\varphi), \sin (\varphi)$, 0$]$, in order to break the geometric symmetry of the polygons and show the electron arrangements within samples.

The single-particle eigenvalues and eigenstates are obtained with a discretization method based on a polar grid. To construct the model of a polygonal ring we first define a circular disk [53] on which we superimpose polygonal constraints and further reduce the grid to the sites situated between the boundaries, Fig. 1. In the position representation the Hilbert space associated with the polar grid is spanned by vectors $|q\rangle \equiv|k j \sigma\rangle$, where the two first integer numbers represent the discretized radial $\left(r_{k}\right)$ and angular $\left(\phi_{j}\right)$ coordinates, respectively, and $\sigma= \pm 1$ stands for the spin projection in the growth $(z)$ direction. The numerical diagonalization of the Hamiltonian (1) allows us to obtain, separately for each band i, the single-particle eigenvalues $E_{\mathrm{i}, a}(a=1,2,3, \ldots)$ and eigenvectors $\left|\Psi_{\mathrm{i}, a}\right\rangle$.

In the second quantization approach the multi-electron Hamiltonian of interacting electrons is

$$
\hat{H}=\sum_{a} E_{a} a_{a}^{\dagger} a_{a}+\frac{1}{2} \sum_{a b c d} V_{a b c d} a_{a}^{\dagger} a_{b}^{\dagger} a_{d} a_{c}
$$

where $E_{a} \equiv E_{\mathrm{i} a}$ are the single-particle eigenvalues, $a_{a}^{\dagger}$ and $a_{a}$ are the electronic creation and annihilation operators acting on particles in the single-particle eigenstate $\left|\Psi_{a}\right\rangle \equiv\left|\Psi_{\mathrm{i}, a}\right\rangle$ and $V_{a b c d}$ are the Coulomb integrals.

In the electron-hole approach the (single) exciton Hamiltonian is given by

$$
H_{\mathrm{e}-\mathrm{h}}=H_{\mathrm{e}}+H_{\mathrm{h}}-\frac{1}{2} V
$$

where $H_{\mathrm{e}}$ is the electronic Hamiltonian which refers to an electron in the conduction band. This is the Hamiltonian (1) for $\mathrm{i}=\mathrm{c}, H_{\mathrm{e}}=H_{\mathrm{c}}$. The second element describes a hole in the valence band and is also derived from the formula (1) but this time the effective mass $m_{\mathrm{i}}^{*}$ is replaced by $-m_{\mathrm{v}}^{*}, H_{\mathrm{h}}=H_{\mathrm{v}}\left(m_{\mathrm{i}}^{*}=-m_{\mathrm{v}}^{*}\right)$. The single-particle eigenvalue problems,

$$
\begin{aligned}
H_{\mathrm{e}}\left|\Psi_{\mathrm{e}}\right\rangle & =\mathcal{E}_{\mathrm{e}}\left|\Psi_{\mathrm{e}}\right\rangle, \\
H_{\mathrm{h}}\left|\Psi_{\mathrm{h}}\right\rangle & =\mathcal{E}_{\mathrm{h}}\left|\Psi_{\mathrm{h}}\right\rangle,
\end{aligned}
$$

are solved with the discretization method. The third element, $V$, stands for the attractive interaction between the electron and the hole.

The detailed description of the computational methods can be found in the Appendix. 

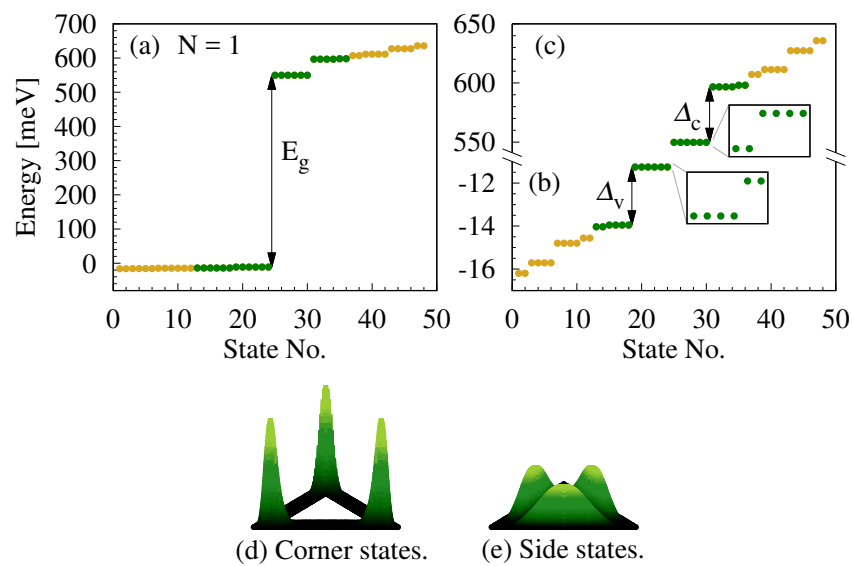

FIG. 2. (a) Single-particle energy levels close to the band edges for a triangular shell. The separation between the lowest conduction and highest valence levels $\left(E_{\mathrm{g}}=561 \mathrm{meV}\right)$ is considerably increased with respect to the bulk value $\left(E_{\mathrm{b}}=354 \mathrm{meV}\right)$ due to the confinement. (b) and (c) zoomed valence and conduction levels. The arrows indicate the splittings between corner and side spin-orbital states in the two bands $\left(\Delta_{\mathrm{v}}=2.7 \mathrm{meV}\right.$ and $\left.\Delta_{\mathrm{c}}=47 \mathrm{meV}\right)$. Insets to both figures show the degeneracy of corner spin-orbital states in each band. (d) and (e) electron localizations of the single-particle states used to construct the multi-electron Hamiltonian [green circles in Figs. (a-c)]. The 6 lowest conduction states and 6 highest valence states are of corner type (d), while the groups of 6 states above and below the $\Delta_{\mathrm{c}}$ and $\Delta_{\mathrm{v}}$, respectively, are localized in the sides (e).

\section{RESULTS}

Below we present the results obtained for the properties of excitons confined in polygonal shells achieved with a discretization method where the samples were approximated by a grid consisting of the number of site points which varies between 8145 and 13380 depending on the polygon shape. For the studied triangular cross section the side thickness $(d)$ and the external radius $\left(R_{\text {ext }}\right)$ equal 8 and $50 \mathrm{~nm}$, respectively. In the case of square and hexagonal samples we show results obtained for the same parameters and for reduced values of $d$ or $d$ and $R_{\text {ext }}$. The numerical calculations were performed for InAs material parameters which are $m_{\mathrm{c}}^{*}=0.023 m_{\mathrm{e}}, m_{\mathrm{v}}^{*}=-0.4 m_{\mathrm{e}}, g_{\mathrm{c}}^{*}=g_{\mathrm{v}}^{*}=-15, \epsilon=15$, and $E_{\mathrm{b}}=354 \mathrm{meV}$. The values of the $g$ factors are used here as test values. The radial confinement in the core-shell heterostructure definitely has an influence on these parameters and we are not aware of accurate data. However, the present results are not significantly sensitive to the $g$ factor of electrons or holes, since we consider only a weak magnetic field, in order to lift the spin degeneracy. To test that we also varied $g_{v}^{*}$ by $\pm 50 \%$ and found no significant effect on the results shown. To construct either the multi-electron interacting Hamiltonian (2) or the exciton Hamiltonian (3) we truncate the single-particle spectrum to $4 p$ valence and $4 p$ conduction states, where $p$ is the number of vertices, and solve the eigenvalue problem by diagonalizing both Hamiltonians (Eqs. 2 and 3) in the bases built of those $8 p$ states. In this way we include in the model the corner and side states from both bands in order to describe corner and side localization of electrons or vacancies.

The polygonal geometry of quantum rings (shell's cross sections) implies unique physical properties such as specific energy structures and non-uniform particle distributions along the ring's perimeter. For symmetric polygons single-particle energy levels are two- and 

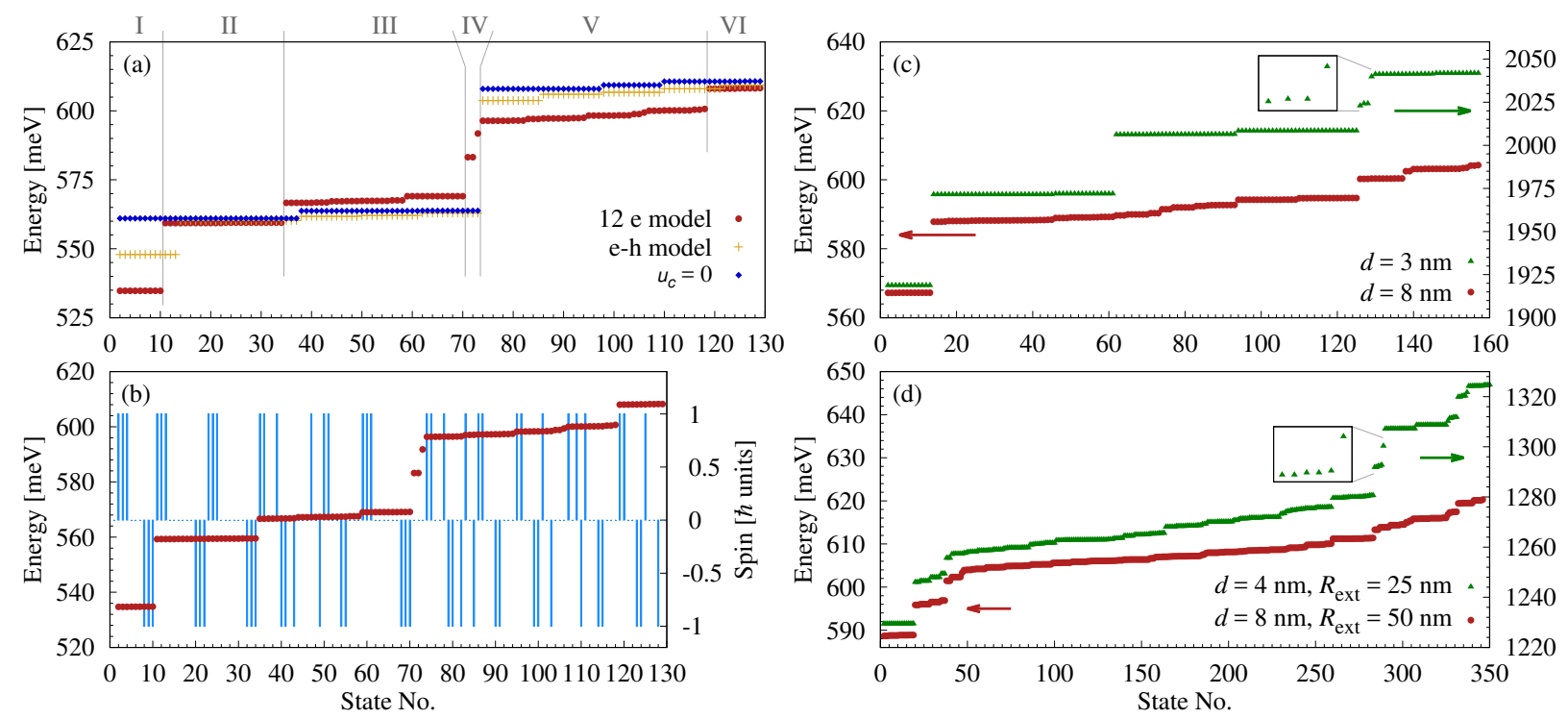

FIG. 3. (a) and (b) Excitonic energy levels for a symmetric triangular shell. (a) Results for non-interacting electrons (blue diamonds) and for Coulomb interacting particles achieved with the electron-hole model (yellow crosses) and with the multi-electron model (red circles). This later spectrum is divided in six zones, I to VI, see text. (b) Excitonic energy levels in the presence of an external (static) magnetic field of $B=50 \mathrm{mT}$ (red circles) and the corresponding $z$ th component of spin (light blue bars). (c) Excitonic energy levels for two symmetric square shells of different side thickness $(d)$. The shells are restricted externally by the same external radius $\left(R_{\text {ext }}=50 \mathrm{~nm}\right)$. (d) Excitonic energy levels for two symmetric hexagonal shells. The shells differ in both side thickness $(d)$ and external radius $\left(R_{\text {ext }}\right)$. The green and red arrows in Figs. (c) and (d) indicate the relevant axes.

fourfold degenerate due to spin only or spin and orbital momentum, respectively, Figs. 2(a)$2(\mathrm{c})$. The energy spectra are arranged in groups of $2 p$ spin-orbital states, corresponding to similar electron distributions. In particular, the number of localization peaks formed on each ring's side is conserved within each group and differs by one between adjacent sets of $2 p$ states, Figs. 2(d) and 2(e) [18]. The exception are the lowest group of conduction band states and the highest valence band states. Both these groups consist of cornerlocalized states, i.e., electrons having the corresponding energies occupy the areas between the sample boundaries and, for sufficiently thin rings, are depleted from the sides, Figs. $2(d)$. The probability distributions associated with the groups above and below the corner domain form one maximum in each side, Figs. 2(e). The energy gaps between corner and side states, $\Delta_{\mathrm{v}}$ and $\Delta_{\mathrm{c}}$, for valence and conduction bands, respectively, are the largest gaps within each band and in both cases exceed the energy dispersion of corner states by few orders of magnitude. Due to the large effective mass, the splittings between adjacent valence levels are much smaller than within the conduction band, and thus $\Delta_{\mathrm{V}}$ is over one order of magnitude smaller than $\Delta_{\mathrm{c}}$, Figs. 2(b) and 2(c).

We describe the exciton confined in a triangular shell with two methods: a purely electronic one and an electron-hole method. In the case of the multi-electron approach, the ground state and the zero energy level correspond to the fully occupied valence band. The exciton is created when one of the electrons is excited to the conduction band leaving at the same time a vacancy in the valence band. To describe the top of the valence band 


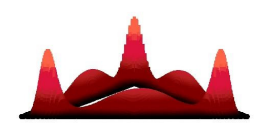

(a) Zone I.

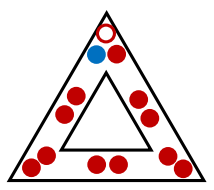

(b) Zone II.
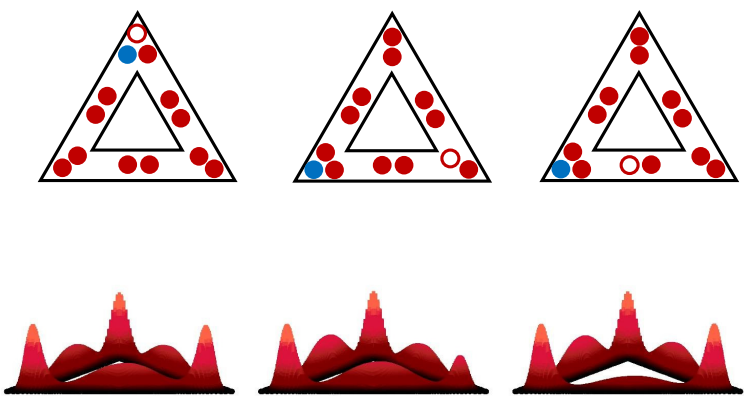

(d) Zone IV
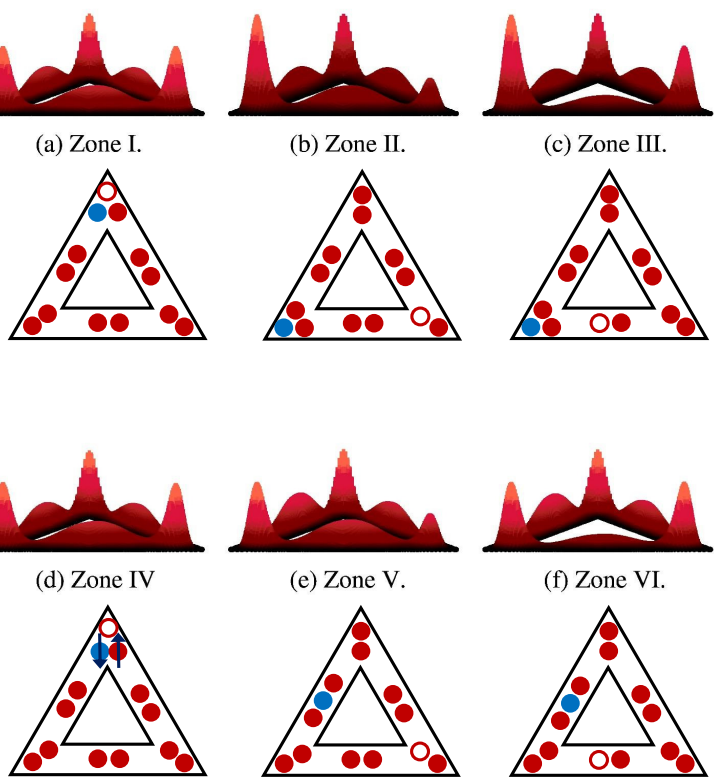

(c) Zone III.

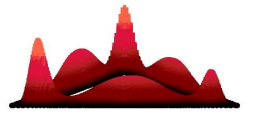

(e) Zone V.
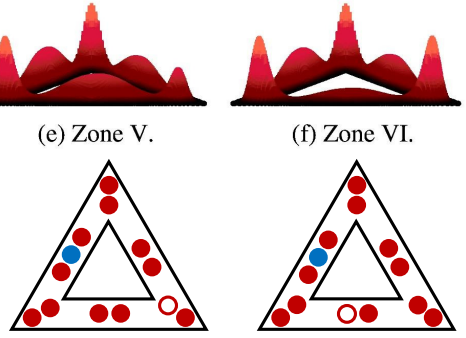

(f) Zone VI.

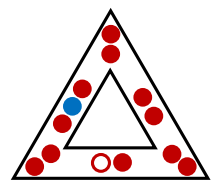

FIG. 4. Probability distributions associated with the representative excitonic states of a triangular shell with broken symmetry obtained with the multi-electron method (upper) and the corresponding electron arrangements for each spectral zone indicated in Fig. 3(a). The filled red circles stand for the valence electrons, the red empty circle represents the vacancy in the valence band, while the blue circle indicates the position of the conduction electron. Arrows in Fig. (d) stand for the spin singlet configuration.

we restrict the system to 12 electrons, i.e., we assume that only electrons localized in the corners or along the whole side length [as in Figs. 2(d) and 2(e)] may be shifted above the semiconductor band gap $\left(E_{\mathrm{b}}\right)$. This means that our ground state is constructed with the 12 highest valence states, i.e., 6 corner and 6 side spin-orbital states. The restriction on the numbers of single-particle states and electrons is necessary for taking into account the Coulomb interaction in a reasonable configuration-interaction space.

In the electron-hole model an exciton is defined as a pair consisting of a conduction electron and a hole in the valence band. Originally, in this model the ground state corresponds to the exciton of the lowest energy, but to be able to compare results obtained with the two methods we assumed that, like in the case of the electronic model, the ground state and zero energy level correspond to the absence of excitons, and thus each excited state is associated with the existence of an electron-hole pair.

If Coulomb interaction is not taken into consideration then both methods provide the same results. As can be seen in Fig. 3(a) (blue diamonds) the excitonic states are split into two nearly dispersionless groups separated approximately by $\Delta_{\mathrm{c}}$, i.e., they differ by the localization of the conduction electron which may occupy corner or side areas. Although the energy gap $\Delta_{\mathrm{V}}$ is much smaller than $\Delta_{\mathrm{c}}$, the splitting it causes in the middle of the lower band (between states 37 and 38) is visible. This gap separates the excitonic states into purely corner ones, which consist of the conduction electron and the hole or vacancy both localized on corners, and those created by an excitation of the electrons from a side to a corner area. In the absence of Coulomb interaction the particle distribution does not depend on the spatial separations, but only on the single-particle energies and their localizations. This allows for the lowest exciton state to be associated with the electron distribution which 
contains 3 electrons ( 2 in the valence and 1 in the conduction band) accumulated in the same corner.

The Coulomb interaction included within the electron-hole model shifts the energy levels to lower energies with respect to the non-interacting case [yellow crosses in Fig. 3(a)]. The largest contribution due to the Coulomb attraction is to excitons composed of nearby electrons and holes, i.e., when both particles occupy the same corner area. The group of 12 such states is clearly separated from the lower band and pulled to much lower energies. The following 24 states corresponding to electrons and holes localized in different corners are only slightly affected by the Coulomb interaction. The effect increases with decreasing spatial separation of the particles, and thus becomes more pronounced for excitons distributed between corner and side areas $(38 \leq \alpha \leq 73)$. Although this model was successfully used to explain properties of excitons in various nanostructures, it did not allow us to reproduce all features obtained with the multi-electron approach. Our implementation of the electronhole model takes into account only two (distinct) particles, which is not enough to properly describe arrangements with 3 close by electrons, especially accumulated in one corner area, and subjected to Pauli's principle.

The energy levels calculated with the multi-electron (configuration-interaction) method are shown in Fig. 3(a) with red circles. The probability distributions for symmetric polygons always obey the geometric symmetries. Therefore, in order to identify the number of electrons occupying particular corners or side areas, we perturb the system symmetry in such a way that every single-particle state is localized on one corner or mostly on one side. For the triangular case we use a relatively weak electric field of $0.22 \mathrm{mV} / R_{\text {ext }}$, parallel to one of the sides, which considerably rearranges the electron localization and only slightly affects the energy levels, but enough to remove the orbital degeneracies [54]. To also lift the spin degeneracies we include an external magnetic field but we keep it weak enough not to change the particle localization.

The lowest group of 9 states [zone I in Fig. 3(a), $2 \leq \alpha \leq 10$ ] is associated with almost symmetric probability distributions even though the single-particle probability distributions form single peaks on corner and side areas, Fig. 4(a). This, together with a very small energy dispersion $\left(E_{10}-E_{2}=0.0011 \mathrm{meV}\right)$, suggests that the conduction electron and the vacancy are localized on the same corner.

The second quasidegenerate level consists of 24 states [zone II in Fig. 3(a), $11 \leq \alpha \leq 34$ ] spread within an interval of $0.16 \mathrm{meV}$. The corresponding probability distributions form 3 equal maxima on the sides and 3 peaks of different height in the areas between the internal and external boundaries, which clearly indicates that there are 3 electrons accumulated in 1 corner area, 2 particles close to the second vertex and 1 electron on the third corner, Fig. 4(b). Since at most 2 valence electrons may stay so close to each other, the third particle in the corner must be the electron excited to the conduction band, while the single valence electron is paired with the vacancy. Due to the few-particle interaction the splitting between the two lowest groups of states is much larger than the obtained with the electronhole model. Another discrepancy between the two models, although related to the previous one, is in the estimation of the binding energy. In the electron-hole model the exciton binding energy is defined as the difference between the energies of the non-interacting exciton and the Coulomb interacting one, both with the lowest energies (i.e. the 'ground' excitons). For the $8 \mathrm{~nm}$ triangular shell it gives a value of $13 \mathrm{meV}$, as can be seen in the Fig. 3(a) by comparing the first blue and yellow points, respectively. For the multi-electron approach it is more difficult to define the binding energy. It should be defined as the energy needed 
to disassemble the exciton, i.e., move the conduction electron somewhere where no vacancy exists. In order to do that, we calculated the ground state of the system with the fully occupied valence band plus one electron in the conduction band, from which we subtracted the energy of the excitonic state, but also the 'charging energy' of the valence band. This 'charging energy' is the energy difference between the fully occupied valence band and the configuration with one vacancy. With this procedure, the estimated binding energy (for the studied triangular shell) is $26 \mathrm{meV}$.

The energy dispersion of the third group of states [zone III in Fig. 3(a), $35 \leq \alpha \leq 70$ ] increases because of side-localized vacancies, Fig. 3(b). As in the case of the splitting between the 2 lowest quasidegenerate levels, also the energy separation of the second and the third group of states increases with the number of interacting electrons taken into account.

The 3 states $[\alpha=71,72$, and 73, zone IV in Fig. 3(a)] in the gap separating energies of excitons consisting of a conduction electron in a corner area or on a side are obtained only with the multi-electron method. Like the 9 lowest excitonic states, these 3 states are associated with symmetric probability distributions, Fig. 4(d). To understand why they gained so much more energy than other states of the same localization type we repeated the calculations of energy levels in the presence of a weak external magnetic field $(B=50 \mathrm{mT})$ [red circles in Fig. 3(b)] which lifts the degeneracies, and thus allows to properly identify the spin of each state. The ground state (zero energy level, fully occupied valence band) is non-degenerate and in spin singlet configuration. Although 12 electrons are taken into consideration, there are only 3 possible values of the $z$ th component of the exciton spin: $-\hbar, 0$, and $\hbar$, indicated by the light blue bars in Fig. 3(b), which are characteristic for a pair of $\pm \hbar / 2$ spin particles. Such system may be in one of the triplet $(|1,1\rangle,|1,0\rangle,|1,-1\rangle)$ configurations or in a singlet $(|0,0\rangle)$ state. Due to the system symmetry and equivalent electron distributions, each of the 4 spin configurations is repeated within every group of the same localization type. For example, there are 12 states $[2 \leq \alpha \leq 10$, and $71 \leq \alpha \leq 73$, zones I and IV in Fig. 3(a)] associated with the conduction electron and the vacancy both localized on the same corner. As can be seen in Fig. 3(b), two values of the $z$ th spin component $(-\hbar$ and $\hbar$ ), are repeated 3 times while the third value (0) occurs 6 times within the group, i.e., the whole set of 4 spin configurations is reproduced 3 times. The 9 lower states are in the triplet configurations and form a quasidegenerate level, while the 3 remaining states are moved to much higher energies. The spin singlet configuration allows the particles to approach so close to each other that the contribution due to the Coulomb interaction of symmetrically distributed electrons exceeds even the one corresponding to 3 electrons accumulated in one corner area [zones II and III in Fig. 3(a), $11 \leq \alpha \leq 70$ ]. Two of these excitonic in-gap states are almost degenerate, Fig. 3(b), while the energy of the third state is substantially higher. The degeneracy is rigorous in the absence of the external magnetic field, Fig. 3(b), and it is related to the $\mathrm{CW}$ and $\mathrm{CCW}$ rotational symmetries of the polygon. As already mentioned these are the orbital degeneracies of the single-particle states[18] which have also been obtained for few-electron states in a single (conduction) band [50].

Higher states correspond to excitons consisting of conduction electrons occupying sides and vacancies localized in corners, Fig. 4(e), or on side areas, Fig. 4(f). The interaction between larger number of electrons resulted in increased splittings between states associated with different particle arrangement for lower states (up to the state No. 70). The gap that originates from $\Delta_{c}$ is affected in the opposite way and considerably shrinks with respect to the non-interacting case and the results achieved with the electron-hole model. If the 3 states in the gap are not taken into consideration, the states approaching the gap from 
lower and higher energies differ by the spatial separation of electrons. In particular, the lower states correspond to arrangements that include 3 electrons accumulated in a small corner area, Fig. 4(c), while the higher states correspond to conduction electrons sharing side areas together with 2 other (valence) electrons, Fig. 4(e), i.e., the set of 3 electrons is distributed on a much larger area than in the former case. This results in a much smaller contribution due to Coulomb repulsion to these states than to the states below the gap, and thus considerable reduction of the splitting between the two groups [zones III and V in Fig. $3(\mathrm{a})]$.

The triangular quantum rings have the lowest possible number of vertices, the sharpest corners and the longest edges. The combination of these features results in sharp and well separated corner peaks, Fig. 2(d). Moreover, the ratio between the internal edge length and the side thickness $(d)$ is also the largest and thus the side maxima are strongly elongated, Fig. 2(e). The considerable difference between corner and side areas, and thus between the probability distributions forming maxima in these two regions, results in large energy gap between corner and side states. All these effects diminish with increasing the number of vertices. In particular, the corner localization softens and the probability distributions, which still form maxima in the corner areas, acquire long tails which penetrate into the sides and overlap. The edges are also shortened, and thus the ratio between the side edge and thickness decreases which results in the sharpening of the side-localized maxima, i.e., the peaks formed in the sides start to resemble the ones in the corners, and thus energy separation between the two groups of states decreases, even to values comparable with other splittings [18].

Although the energy gap separating corner from side states in the conduction band is still considerably large and for the $8 \mathrm{~nm}$ thick square shell equals $20.6 \mathrm{meV}$, the splitting between excitonic states containing the conduction electron in the corner or in the side area is considerably reduced due to the Coulomb repulsion and contrary to the triangular case it is not broken by high energy corner states, Fig. 3(c) (red circles). Four corners of a square shell allow for 16 states corresponding to the conduction electron and the vacancy both localized on the same corner. As seen in Fig. 3(c) only 12 states $(2 \leq \alpha \leq 13)$ of this type are separated from the spectrum and shifted to lower energies. Similarly to the triangular shell, the 4 remaining states acquired much more energy, but due to the relatively small energy splitting between the lowest states and the states corresponding to conduction electrons occupying side areas, those 4 purely corner excitonic states mix with the latter states.

The separation between corner and side states increases with decreasing the side thickness [19]. Therefore, to considerably separate the two types of excitonic states and to keep the high-energy corner states within the gap we reduced the side thickness to $3 \mathrm{~nm}$, Fig. 3(c) (green triangles). The 4 in-gap states reproduce the degeneracy of single-particle corner states with respect to the spin [18], i.e., the lowest and highest states ( $\alpha=126$ and 129) are non-degenerate, while the middle states are twofold degenerate. Contrary to the $8 \mathrm{~nm}$ triangular shell, Figs. 3(a) and 3(b), for the studied square structures the largest energy splitting stems not from the gap between corner and side states $\left(\Delta_{\mathrm{c}}\right)$, but is a result of a large increase of the Coulomb interaction due to the change of the number of electrons in particular corners. As seen in Fig. 3(c), this splitting is much larger for the thinner shell. The areas between the internal and external boundaries shrink with the thickness, and thus the corner localized electrons are accumulated on much smaller areas which results in strongly increased Coulomb interaction for the thinner shells. Since 4 corners of a square 
ring allow for more combinations of electron distribution, the energy structure is richer than for the triangular shell. The purely corner states, which are associated with non-symmetric electron arrangements, form two close by levels corresponding to the conduction electron and the vacancy occupying neighboring $(14 \leq \alpha \leq 45)$ and alternating $(46 \leq \alpha \leq 61)$ corners. The small difference between these two levels shows that Coulomb interaction between electrons localized in different corners is negligibly small, at least for the analyzed edge length. The following 64 states correspond to vacancies localized on sides and the conduction electron in one of the corners, i.e., there are 3 pairs of electrons in 3 corners while the fourth corner is occupied by 3 particles. The thinner the shell is, the narrower the effective quantum wells formed in corner areas are, and thus the higher the energy levels are. This results in a strong increment of energies with the decrease of side thickness shown on the two vertical axes of Fig. 3(c).

As in the cases of the two already analyzed polygons, also for the hexagonal shells the lowest states are separated from the spectrum, Fig. 3(d). The dispersion of these 18 states is still very small but larger than for shells with lower number of vertices. The Coulomb induced splittings of the lowest groups of states are comparable for equally thick square and triangular shells, but this does not hold for the hexagonal samples. In fact, the corner localization softens with increasing the number of corners, and, for the $8 \mathrm{~nm}$ thick hexagonal shell, the corner peaks penetrate into the sides and overlap. This effectively increases the corner areas and allows for much larger spatial separation between corner-localized electrons which considerably decreases the Coulomb interaction of few particles accumulated in one corner. At the same time, the interaction between electrons occupying neighboring corner and side areas increases considerably, as well as, to a lesser extent, the interaction of electrons in consecutive corners. Moreover, for hexagonal shells the overlap of single-particle states strongly depends on the energies, and thus also the contributions due to the Coulomb interaction may differ even within the group of states associated with similar particle arrangements. As a result some of the states corresponding to unequal electron distributions $(20 \leq \alpha \leq 47)$ are pulled towards lower energies. The effect is amplified by the reduction of the distances between the vertices and the possibility of different distributions of the conduction electron and the vacancy within the cross section. If the external radius and side thickness are not changed, then the energy gap separating corner from side conduction states of a hexagonal shell is much smaller than the one of square sample and for the $8 \mathrm{~nm}$ thick shell it hardly allows to separate the two types of excitonic states. Here the dominant splittings are purely of Coulomb origin, Fig. 3(d) (red circles).

The separations between corner and side states increase with decreasing the aspect ratio $\left(d / R_{\text {ext }}\right)[19]$ and external radius $\left(R_{\text {ext }}\right)$. To move the 6 high-energy corner states below states corresponding to conduction electrons occupying side areas we had to decrease both, the side thickness $(d)$ and the diameter $\left(2 R_{\text {ext }}\right)$ to 4 and $50 \mathrm{~nm}$, respectively. As shown in Fig. 3(d) (green triangles) such a shell provides sufficiently large gap which can host the whole set of 6 states in the spin singlet configuration for which the conduction electron and the vacancy are localized in the same corner area.

\section{EXCITATIONS LIFETIME}

In the electron-hole model the exciton lifetime is determined by the overlap of the electron and hole wavefunctions, i.e., it depends on the localization of the two particles. Similarly, in the multi-electron method it is defined by the overlap of the initial and final wavefunctions 


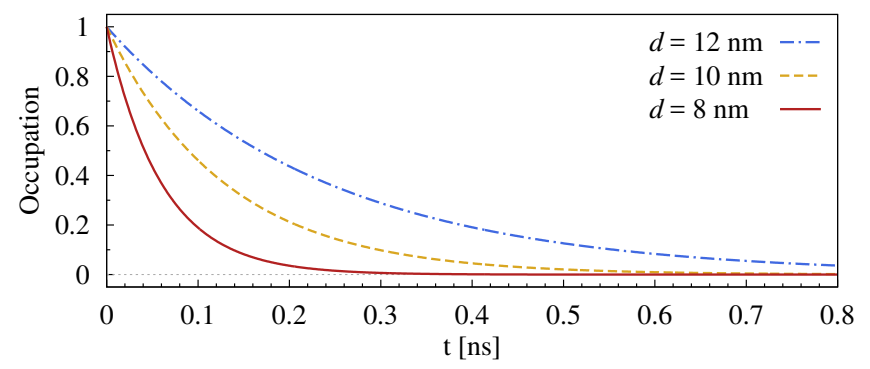

FIG. 5. Lifetime of in-gap excitons $(\alpha=71$ and 72) for few values of the side thickness $(d)$.

which also reduces to the dependence on the distribution of the conduction electron and the vacancy within the shell. In this approach the ground state corresponds to the fully occupied valence band, and thus to almost symmetrically distributed electrons. In the triangular geometry this means that there are two electrons in each corner area and on every side. Therefore for the examples shown in Figs. 3(a) and 3(b) the ground state has the largest overlap with the excitonic states of zones I and IV [Figs. 3(a) and 4] and with some higher states corresponding to conduction electron and the vacancy both localized on the same side, in zone VI. For all of the other states the conduction electron and the vacancy are well separated, i.e., their localization areas do not overlap, Fig. 4. Consequently, the recombination process is blocked for states belonging to zones II, III, and V. Our results are in agreement with the selection rules identified by other authors for hexagonal InP nanowires. [55]

Furthermore, in our model we do not take into account spin-orbit interaction [56], and thus the transitions are restricted to those which conserve spin. Since the ground state is in spin singlet configuration, none of the 9 states from zone I may release a photon. In fact, spin-orbit coupling is effective in InAs tubular structures that are grown along a crystallographic direction different from [111] or with cross sections lacking inversion symmetry in one direction, as the triangular-shell samples we address. In this case, the mixing of spin and orbital degrees of freedom will break several energy degeneracies of the multi-electron spectra, as the ones reported in Fig. 3. On the one hand, this effect is non-negligible since the typical diameter of our samples, according to recent experiments [57], is of the same order as the spin-orbit length in InAs nanowires. On the other hand, spin-orbit coupling is expected to be small, compared to the energy gaps of tens of meV which we obtain in multi-particle spectra, and the intensities of optical transitions forbidden by spin selection rules in our calculations will be considerably weaker than the spin-conserving ones. A quantitative estimation of spin-orbit coupling in core-shell nanowires is beyond the scope of this work $[58,59]$.

Now, we focus on the three excitonic states of zone IV, which correspond to spin singlet configuration, with the conduction electron and vacancy localized on the same corner, Fig. $4(\mathrm{~d})$. We can imagine two possible photon polarizations: within the $x-y$ plane, i.e., transversal to the nanowire, or in the $z$ direction, i.e., longitudinal to the nanowire.

In the case of transversal polarization the transition from the highest of these 3 states is also inhibited. The reason is that this state and the ground state have no orbital momentum to compensate that of the photon. As a result the only optically active states are the lowest pair of states ( $\alpha=71$ and 72), with opposite chirality, which in the absence of a symmetry breaking field are degenerate. In Fig. 5 we compare the lifetimes of these states for different shell thicknesses. As can be seen, the decay time considerably varies within a 
nanosecond range and increases with the shell thickness. The energy of the lowest excitonic state is shifted to lower energies and the splittings between adjacent levels shrink for thicker rings [19]. When increasing the side thickness, the corner areas increase as well, i.e., the areas where the two electrons are localized and it results in a weaker Coulomb interaction. Consequently, the energy of the excitonic in-gap states decreases, and thus their lifetime, which is proportional to the inverse of the energy, increases.

In the case of longitudinal polarization, the behavior of the states in zone IV is reversed, and the in-gap state with the highest energy becomes the only one optically active. In fact, the longitudinal-polarized photon couples to the motion of the electron in the $z$ direction, whereas the transverse modes of the excited and ground state have to match.

\section{CONCLUSIONS}

In summary, we studied the properties of excitons confined in triangular, square and hexagonal shells. We compared the multi-electron and electron-hole models and we showed that the former one allows to find more features, especially those related to the few-electron Coulomb effects consistent with the Pauli principle and spin. Using the multi-electron method we showed that the states corresponding to the same or similar electron distributions are arranged into quasidegenerate levels. The exception are the states associated with both the conduction electron and the vacancy localized on the same corner. Only a fraction of these states ( $3 p$ states) in the spin triplet configuration forms the lowest, nearly dispersionless, level while the remaining states in the spin singlet configuration are shifted to much higher energies. The number of such states equals the number of corners and the set reproduces the degeneracy of single-particle corner states with respect to the spin. For most of the samples these purely corner states mix with states corresponding to conduction electrons localized on the sides. However, if the samples are narrow and the external radii are small, i.e., if the gaps between single-particle corner and side states in the conduction band are sufficiently large, these states fit into the interval that separates excitonic states with the conduction electron occupying a corner area or localized on a side and form distinct excitonic in-gap states.

In the case of triangular shell, the largest gap within the energy spectrum originates from the separation of single-particle corner and side states, but for square and hexagonal shells the Coulomb-induced splitting between the two lowest levels becomes the dominant one.

We also studied the recombination process of an exciton confined in a triangular shell and we showed that most of the excitonic states are dark. The only optically active states are those for which the conduction electron and the vacancy are localized on the same area. The allowed transitions have to conserve spin, i.e., only the states in the spin singlet configuration may decay. Moreover the initial and final states have to correspond to different chirality. These selection rules reduce the number of states which easily recombine to the in-gap states. 


\section{Appendix: Computational methods}

\section{Single-particle Hamiltonian matrix elements}

For convenience we split the single-particle Hamiltonian (Eq. 3 in the main text) into four terms

$$
H_{\mathrm{i}}=H_{\mathrm{i}}^{\mathrm{K}}+H_{\mathrm{i}}^{\mathrm{B}}+H_{\mathrm{i}}^{\mathrm{Z}}+H_{\mathrm{i}}^{\mathrm{E}}
$$

and calculate matrix elements of each component separately in the polar basis $\{|k j \sigma\rangle\}$, where $k$ and $j$ are the discretized radial $\left(r_{k}\right)$ and angular $\left(\phi_{j}\right)$ coordinates, respectively, and $\sigma= \pm 1$ stands for the spin projection in the $(z)$ direction.

The first element, the kinetic Hamiltonian in the absence of magnetic field, is given by

$$
\begin{aligned}
\left\langle k j \sigma\left|H_{\mathrm{i}}^{\mathrm{K}}\right| k^{\prime} j^{\prime} \sigma^{\prime}\right\rangle= & T_{\mathrm{i}} \delta_{\sigma, \sigma^{\prime}}\left[t_{r}\left(\delta_{k, k^{\prime}}-\delta_{k, k^{\prime}+1}\right) \delta_{j, j^{\prime}}+t_{\phi} \delta_{k, k^{\prime}}\left(\delta_{j, j^{\prime}}-\delta_{j, j^{\prime}+1}\right)+\text { H.c. }\right] \\
& +E_{\mathrm{b}} \delta_{\mathrm{el}, \mathrm{i}} \delta_{k, k^{\prime}} \delta_{j, j^{\prime}} \delta_{\sigma, \sigma^{\prime}},
\end{aligned}
$$

where $T_{\mathrm{i}}=\hbar^{2} /\left(2 m_{\mathrm{i}}^{*} R_{\text {ext }}^{2}\right)$ is a reference energy for the valence $(\mathrm{i}=\mathrm{v})$ and conduction $(\mathrm{i}=\mathrm{c})$ bands, respectively, and $m_{\mathrm{v}, \mathrm{c}}^{*}$ are the corresponding effective masses. $R_{\text {ext }}$ is the external radius of the polar grid and also of the polygonal shell. $t_{r}=\left(R_{\text {ext }} / \delta r\right)^{2}, t_{\phi}=\left[R_{\text {ext }} /\left(r_{k} \delta \phi\right)\right]^{2}$, $\delta r$ is the distance between neighboring sites with the same angle, $\delta \phi$ is the angle between adjacent sites with the same radius and $E_{\mathrm{b}}$ is the semiconductor (bulk) energy gap.

The second element is the correction to the kinetic energy due to the magnetic field $(B)$

$$
\left\langle k j \sigma\left|H_{\mathrm{i}}^{\mathrm{B}}\right| k^{\prime} j^{\prime} \sigma^{\prime}\right\rangle=T_{\mathrm{i}} \delta_{\sigma, \sigma^{\prime}} \delta_{k, k^{\prime}}\left[\frac{1}{2} t_{\mathrm{B}-\mathrm{i}}^{2}\left(\frac{r_{k}}{4 R_{\mathrm{ext}}}\right)^{2} \delta_{j, j^{\prime}}-t_{\mathrm{B}-\mathrm{i}} \frac{i}{4 \delta \phi} \delta_{j, j^{\prime}+1}+\text { H.c. }\right],
$$

where $t_{\mathrm{B}-\mathrm{i}}=\hbar e B / m_{\mathrm{i}}^{*} T_{\mathrm{i}}$ is the cyclotron energy in the units of $T_{\mathrm{i}}$.

The third term, the Zeeman part, is given by

$$
\left\langle k j \sigma\left|H_{\mathrm{i}}^{\mathrm{Z}}\right| k^{\prime} j^{\prime} \sigma^{\prime}\right\rangle=\frac{1}{2} T_{\mathrm{i}} t_{\mathrm{B}-\mathrm{i}} \gamma_{\mathrm{i}}\left(\sigma_{z}\right)_{\sigma, \sigma^{\prime}} \delta_{k, k^{\prime}} \delta_{j, j^{\prime}}
$$

here $\gamma_{\mathrm{i}}=g_{\mathrm{i}}^{*} m_{\mathrm{i}}^{*} / 2 m_{\mathrm{e}}$ is the ratio between the Zeeman gap and the cyclotron energy, and $m_{e}$ is the free electron mass.

Finally, the last term stands for the contribution due to the electric field

$$
\left\langle k j \sigma\left|H_{\mathrm{i}}^{\mathrm{E}}\right| k^{\prime} j^{\prime} \sigma^{\prime}\right\rangle=-e E r_{k} R_{\mathrm{ext}} \delta_{k, k^{\prime}} \delta_{j, j^{\prime}} \delta_{\sigma, \sigma^{\prime}}\left[\cos (\varphi) \cos \left(\phi_{\mathrm{i}}\right)+\sin (\varphi) \sin \left(\phi_{\mathrm{i}}\right)\right],
$$

where $E$ is the amplitude of the field and $\varphi$ the angle it make with the $x$ axis.

The numerical diagonalization of the matrices consisting of the elements $\left\langle k j \sigma\left|H_{\mathrm{v}, \mathrm{c}}\right| k^{\prime} j^{\prime} \sigma^{\prime}\right\rangle$ provides the single-particle eigenvalues $E_{\mathrm{i}, a}(a=1,2,3, \ldots)$ and eigenvectors $\left|\Psi_{\mathrm{i}, a}\right\rangle$ in the basis $\{|k, j, \sigma\rangle\}$, i.e., $\left|\Psi_{\mathrm{i}, a}\right\rangle=\sum_{q} \psi_{\mathrm{i}}(q, a)|q\rangle$, where $|q\rangle \equiv|k, j, \sigma\rangle$ and $\psi_{\mathrm{i}}(q, a)$ are the amplitudes of the eigenvectors in the polar basis $|q\rangle$.

\section{Reduction of the basis for the multi-electron approach}

The multi-electron eigenvalues $\mathcal{E}_{\alpha}(\alpha=1,2,3, \ldots)$ and eigenstates $\left|\Phi_{\alpha}\right\rangle$ of the Hamiltonian (Eq. 2 in the main text) are obtained by (exact) numerical diagonalization of $\hat{H}$ in 
a truncated Fock space constructed of $N_{\mathrm{v}}$ valence and $N_{\mathrm{c}}$ conduction band single-particle eigenstates. If there are $N$ electrons confined in the system, then the basis consists of $\left(N_{\mathrm{v}}+N_{\mathrm{c}}\right) ! /\left[N !\left(N_{\mathrm{v}}+N_{\mathrm{c}}-N\right)\right.$ ! $]$ eigenstates of the non-interacting system (Slater determinants in 'bit string' representation ) of the form

$$
|\mu\rangle=\left|v_{1}^{\mu} v_{2}^{\mu} \cdots v_{N_{\mathrm{v}}}^{\mu} c_{1}^{\mu} c_{2}^{\mu} \cdots c_{N_{\mathrm{c}}}^{\mu}\right\rangle,
$$

where $v_{a}^{\mu}=\{0,1\}$ and $c_{a}^{\mu}=\{0,1\}$ are the occupation numbers of the single-particle states in the valence $\left(\left|\Psi_{\mathrm{v}, a}\right\rangle\right)$ and the conduction $\left(\left|\Psi_{\mathrm{c}, a}\right\rangle\right)$ bands, respectively. Since we restrict the analysis to single excitons we reduce the basis $|\mu\rangle$ to states for which $\sum_{\mathrm{i}} v_{\mathrm{i}}^{\mu} \geq N_{\mathrm{v}}-1$. The multi-electron eigenstates are linear combinations of these states

$$
\left|\Phi_{\alpha}\right\rangle=\sum_{\mu} \phi(\mu, \alpha)|\mu\rangle
$$

with $\phi(\mu, \alpha)$ being the amplitudes of the multi-electron eigenstates in the basis $|\mu\rangle$. We assume that most of the valence levels are occupied and that electrons may be excited to the conduction band only from few $\left(N_{\mathrm{v}}\right)$ top valence states.

\section{Matrix elements of the electron-hole Hamiltonian}

The matrix elements of the electron-hole Hamiltonian (Eq. 3 in the main text) are calculated in a truncated basis built of $N_{\mathrm{e}}$ electron and $N_{\mathrm{h}}$ hole single-particle states. There are $N_{\mathrm{e}} N_{\mathrm{h}}$ elements of the exciton basis $\{|\mathrm{eh}\rangle\}$ constructed as tensor products of the singleparticle states, $|\mathrm{eh}\rangle=\left|\Psi_{\mathrm{e}}\right\rangle \otimes\left|\Psi_{\mathrm{h}}\right\rangle$. In this basis the exciton Hamiltonian matrix elements are given by

$$
\left\langle\mathrm{eh}\left|H_{\mathrm{e}-\mathrm{h}}\right| \mathrm{e}^{\prime} \mathrm{h}^{\prime}\right\rangle=\left(\mathcal{E}_{\mathrm{e}}+\mathcal{E}_{\mathrm{h}}\right) \delta_{\mathrm{ee}^{\prime}} \delta_{\mathrm{hh}^{\prime}}-\frac{1}{2}\left\langle\mathrm{eh}|V| \mathrm{e}^{\prime} \mathrm{h}^{\prime}\right\rangle
$$

where $\mathcal{E}_{\mathrm{e}, \mathrm{h}}$ are the single-particle eigenvalues of the Hamiltonians $H_{\mathrm{e}, \mathrm{h}}$ (Eqs. 4a and $4 \mathrm{~b}$ in the main text) and the last element stands for the Coulomb integrals

$$
\left\langle\mathrm{eh}|V| \mathrm{e}^{\prime} \mathrm{h}^{\prime}\right\rangle=\frac{e^{2}}{4 \pi \epsilon \epsilon_{0}} \int d \boldsymbol{r}_{\mathrm{e}} d \boldsymbol{r}_{\mathrm{h}} \frac{\varphi_{\mathrm{e}}^{*}\left(\boldsymbol{r}_{\mathrm{e}}\right) \varphi_{\mathrm{h}}^{*}\left(\boldsymbol{r}_{\mathrm{h}}\right) \varphi_{\mathrm{e}^{\prime}}\left(\boldsymbol{r}_{\mathrm{e}}\right) \varphi_{h^{\prime}}\left(\boldsymbol{r}_{\mathrm{h}}\right)}{\left|\boldsymbol{r}_{\mathrm{e}}-\boldsymbol{r}_{\mathrm{h}}\right|}
$$

where $\boldsymbol{r}_{\mathrm{e}, \mathrm{h}}$ and $\varphi_{e^{\prime}, h^{\prime}}\left(\boldsymbol{r}_{\mathrm{e}, \mathrm{h}}\right)$ are the positions and single-particle wavefunctions of the electron and hole, respectively.

\section{Lifetime}

The exciton evolution or the recombination process is described by the master equation in the Markov and rotating-wave approximations derived according to the Weisskopf-Wigner procedure [60, 61],

$$
\dot{\rho}_{\mathrm{e}}=\pi \lambda\left\{\left[\mathcal{T}(t), \rho_{\mathrm{e}} S(t)\right]+\text { H.c. }\right\}
$$


where the coupling constant $\lambda=\left(6 \pi^{2} \hbar \epsilon \epsilon_{0} c^{3}\right)^{-1}$. The operators $\mathcal{T}(t)$ and $S(t)$ depend on the Bohr frequencies $\omega_{\mathrm{nm}}=\left(\mathcal{E}_{\mathrm{m}}-\mathcal{E}_{\mathrm{n}}\right) / \hbar$ and on the sample geometry through the dipole moment matrix elements $\boldsymbol{d}_{\mathrm{nm}}=\sum_{q} \Psi^{\dagger}(q, n) \Psi(q, m) r_{q}\left[\cos \phi_{q}, \sin \phi_{q}\right]$,

$$
\begin{aligned}
& \mathcal{T}(t)=\sum_{\mathrm{nm}} e^{-i \omega_{\mathrm{nm}} t} \boldsymbol{d}_{\mathrm{nm}} a_{\mathrm{n}}^{\dagger} a_{\mathrm{m}}, \\
& S(t)=\sum_{\mathrm{nm}} \omega_{\mathrm{nm}}^{3} e^{i \omega_{\mathrm{nm}} t} \boldsymbol{d}_{\mathrm{nm}} a_{\mathrm{n}}^{\dagger} a_{\mathrm{m}} .
\end{aligned}
$$

\section{ACKNOWLEDGMENTS}

We are thankful to Sigurdur Ingi Erlingsson and Guido Goldoni for helpful comments and discussions. This work was supported by the Icelandic Research Fund.

[1] C. Blömers, T. Rieger, P. Zellekens, F. Haas, M. I. Lepsa, H. Hardtdegen, Ö. Gül, N. Demarina, D. Grützmacher, H. Lüth, and T. Schäpers, Nanotechnology 24, 035203 (2013).

[2] T. Rieger, M. Luysberg, T. Schäpers, D. Grützmacher, and M. I. Lepsa, Nano Letters 12, 5559 (2012).

[3] F. Haas, K. Sladek, A. Winden, M. von der Ahe, T. E. Weirich, T. Rieger, H. Lüth, D. Grützmacher, T. Schäpers, and H. Hardtdegen, Nanotechnology 24, 085603 (2013).

[4] H. Fan, M. Knez, R. Scholz, K. Nielsch, E. Pippel, D. Hesse, U. Gsele, and M. Zacharias, Nanotechnology 17, 5157 (2006).

[5] F. Qian, Y. Li, S. Gradečak, D. Wang, C. J. Barrelet, and C. M. Lieber, Nano Letters 4, 1975 (2004).

[6] F. Qian, S. Gradečak, Y. Li, C.-Y. Wen, and C. M. Lieber, Nano Letters 5, 2287 (2005).

[7] Physica B: Condensed Matter 404, 4933 (2009).

[8] M. Heurlin, T. Stankevič, S. Mickevičius, S. Yngman, D. Lindgren, A. Mikkelsen, R. Feidenhans'l, M. T. Borgstöm, and L. Samuelson, Nano Letters 15, 2462 (2015).

[9] Y. Dong, B. Tian, T. J. Kempa, and C. M. Lieber, Nano Letters 9, 2183 (2009).

[10] X. Yuan, P. Caroff, F. Wang, Y. Guo, Y. Wang, H. E. Jackson, L. M. Smith, H. H. Tan, and C. Jagadish, Adv. Funct. Mater. 25, 5300 (2015).

[11] T. Rieger, D. Grutzmacher, and M. I. Lepsa, Nanoscale 7, 356 (2015).

[12] A. Bertoni, M. Royo, F. Mahawish, and G. Goldoni, Phys. Rev. B 84, 205323 (2011).

[13] M. Royo, A. Bertoni, and G. Goldoni, Phys. Rev. B 87, 115316 (2013).

[14] M. Royo, A. Bertoni, and G. Goldoni, Phys. Rev. B 89, 155416 (2014).

[15] M. Royo, C. Segarra, A. Bertoni, G. Goldoni, and J. Planelles, Phys. Rev. B 91, 115440 (2015).

[16] A. Ballester, J. Planelles, and A. Bertoni, Journal of Applied Physics 112, 104317 (2012).

[17] C. Estarellas and L. Serra, Superlattices and Microstructures 83, 184 (2015).

[18] A. Sitek, L. Serra, V. Gudmundsson, and A. Manolescu, Phys. Rev. B 91, 235429 (2015).

[19] A. Sitek, G. Thorgilsson, V. Gudmundsson, and A. Manolescu, Nanotechnology 27, 225202 (2016).

[20] A. Manolescu, A. Sitek, J. Osca, L. Serra, V. Gudmundsson, and T. D. Stanescu, Phys. Rev. B 96, 125435 (2017). 
[21] F. Boxberg, N. Søndergaard, and H. Q. Xu, Nano Letters 10, 1108 (2010).

[22] R. Peköz, O. B. Malcıŏlu, and J.-Y. Raty, Phys. Rev. B 83, 035317 (2011).

[23] C. Colombo, P. Krogstrup, J. Nygård, M. L. Brongersma, and A. F. i Morral, New Journal of Physics 13, 123026 (2011).

[24] P. Krogstrup, H. I. Jorgensen, M. Heiss, O. Demichel, J. V. Holm, M. Aagesen, J. Nygard, and A. Fontcuberta i Morral, Nature Photonics 7, 306 (2013).

[25] J. Tang, Z. Huo, S. Brittman, H. Gao, and P. Yang, Nature Nanotechnology 6, 568 (2011).

[26] M. Zhang, X. Gao, A. Barra, P. Chang, L. Huang, R. Hellwarth, and J. G. Lu, Materials Letters 140, 59 (2015).

[27] A. Casadei, E. F. Pecora, J. Trevino, C. Forestiere, D. Rffer, E. Russo-Averchi, F. Matteini, G. Tutuncuoglu, M. Heiss, A. Fontcuberta i Morral, and L. Dal Negro, Nano Letters 14, 2271 (2014).

[28] S.-K. Kim, X. Zhang, D. J. Hill, K.-D. Song, J.-S. Park, H.-G. Park, and J. F. Cahoon, Nano Letters 15, 753 (2015).

[29] T. Ozel, G. R. Bourret, A. L. Schmucker, K. A. Brown, and C. A. Mirkin, Advanced Materials 25, 4515 (2013).

[30] D. Saxena, S. Mokkapati, P. Parkinson, N. Jiang, Q. Gao, H. H. Tan, and C. Jagadish, Nat Photon 7, 963 (2013).

[31] T. Stettner, P. Zimmermann, B. Loitsch, M. Döblinger, A. Regler, B. Mayer, J. Winnerl, S. Matich, H. Riedl, M. Kaniber, G. Abstreiter, G. Koblmüller, and J. J. Finley, Applied Physics Letters 108, 011108 (2016).

[32] C. Li, J. B. Wright, S. Liu, P. Lu, J. J. Figiel, B. Leung, W. W. Chow, I. Brener, D. D. Koleske, T.-S. Luk, D. F. Feezell, S. R. J. Brueck, and G. T. Wang, Nano Letters 17, 1049 (2017).

[33] B. Mayer, D. Rudolph, J. Schnell, S. Morkötter, J. Winnerl, J. Treu, K. Müller, G. Bracher, G. Abstreiter, G. Koblmüller, and J. J. Finley, Nature Communications 4, 2931 (2013).

[34] J. R. Riley, S. Padalkar, Q. Li, P. Lu, D. D. Koleske, J. J. Wierer, G. T. Wang, and L. J. Lauhon, Nano Letters 13, 4317 (2013).

[35] K. Tomioka, J. Motohisa, S. Hara, K. Hiruma, and T. Fukui, Nano Letters 10, 1639 (2010).

[36] M. Fickenscher, T. Shi, H. E. Jackson, L. M. Smith, J. M. Yarrison-Rice, C. Zheng, P. Miller, J. Etheridge, B. M. Wong, Q. Gao, S. Deshpande, H. H. Tan, and C. Jagadish, Nano Letters 13, 1016 (2013).

[37] S. Funk, M. Royo, I. Zardo, D. Rudolph, S. Morkötter, B. Mayer, J. Becker, A. Bechtold, S. Matich, M. Döblinger, M. Bichler, G. Koblmüller, J. J. Finley, A. Bertoni, G. Goldoni, and G. Abstreiter, Nano Letters 13, 6189 (2013).

[38] T. Shi, H. E. Jackson, L. M. Smith, N. Jiang, Q. Gao, H. H. Tan, C. Jagadish, C. Zheng, and J. Etheridge, Nano Letters 15, 1876 (2015).

[39] N. Erhard, S. Zenger, S. Morkötter, D. Rudolph, M. Weiss, H. J. Krenner, H. Karl, G. Abstreiter, J. J. Finley, G. Koblmüller, and A. W. Holleitner, Nano Letters 15, 6869 (2015).

[40] M. Weiß, F. J. R. Schülein, J. B. Kinzel, M. Heigl, D. Rudolph, M. Bichler, G. Abstreiter, J. J. Finley, A. Wixforth, G. Koblmüller, and H. J. Krenner, Journal of Physics D: Applied Physics 47, 394011 (2014).

[41] J. B. Kinzel, F. J. R. Schülein, M. Weiß, L. Janker, D. D. Bühler, M. Heigl, D. Rudolph, S. Morkötter, M. Döblinger, M. Bichler, G. Abstreiter, J. J. Finley, A. Wixforth, G. Koblmüller, and H. J. Krenner, ACS Nano 10, 4942 (2016). 
[42] F. Li, Z. Li, L. Tan, Y. Zhou, J. Ma, M. Lysevych, L. Fu, H. H. Tan, and C. Jagadish, Nanotechnology 28, 125702 (2017).

[43] J. Jadczak, P. Plochocka, A. Mitioglu, I. Breslavetz, M. Royo, A. Bertoni, G. Goldoni, T. Smolenski, P. Kossacki, A. Kretinin, H. Shtrikman, and D. K. Maude, Nano Letters 14, 2807 (2014).

[44] M. De Luca, G. Lavenuta, A. Polimeni, S. Rubini, V. Grillo, F. Mura, A. Miriametro, M. Capizzi, and F. Martelli, Phys. Rev. B 87, 235304 (2013).

[45] F. Buscemi, M. Royo, A. Bertoni, and G. Goldoni, Phys. Rev. B 92, 165302 (2015).

[46] G. Ferrari, G. Goldoni, A. Bertoni, G. Cuoghi, and E. Molinari, Nano Letters 9, 1631 (2009).

[47] B. M. Wong, F. Léonard, Q. Li, and G. T. Wang, Nano Letters 11, 3074 (2011).

[48] F. Qian, Y. Li, S. Gradečak, H.-G. Park, Y. Dong, Y. Ding, Z. L. Wang, and C. M. Lieber, Nat Mater 7, 701 (2008).

[49] S. K. Lim, M. Brewster, F. Qian, Y. Li, C. M. Lieber, and S. Gradečak, Nano Letters 9, 3940 (2009).

[50] A. Sitek, M. Țolea, M. Niţă, L. Serra, V. Gudmundsson, and A. Manolescu, Sci. Rep. 7, 40197 (2017).

[51] M.-E. Pistol and C. E. Pryor, Phys. Rev. B 78, 115319 (2008).

[52] A. W. Long and B. M. Wong, AIP Advances 2, 032173 (2012).

[53] C. Daday, A. Manolescu, D. C. Marinescu, and V. Gudmundsson, Phys. Rev. B 84, 115311 (2011).

[54] A. Sitek, M. Urbaneja Torres, K. Torfason, V. Gudmundsson, and A. Manolescu, Proceedings of the 19th International Conference on Transparent Optical Networks (ICTON 2017) (2017).

[55] K. Pemasiri, H. E. Jackson, L. M. Smith, B. M. Wong, S. Paiman, Q. Gao, H. H. Tan, and C. Jagadish, Journal of Applied Physics 117, 194306 (2015).

[56] P. Wójcik, J. Adamowski, M. Wołoszyn, and B. Spisak, Physica E: Low-dimensional Systems and Nanostructures 59, 19 (2014).

[57] F. Krizek, J. E. Sestoft, P. Aseev, S. Marti-Sanchez, S. Vaitiekenas, L. Casparis, S. A. Khan, Y. Liu, T. Stankevic, A. M. Whiticar, A. Fursina, F. Boekhout, R. Koops, E. Uccelli, L. P. Kouwenhoven, C. M. Marcus, J. Arbiol, and P. Krogstrup, arXiv:1802.07808 (2018).

[58] P. Wójcik, A. Bertoni, and G. Goldoni, arXiv:1801.09905 (2018).

[59] T. Campos, P. E. F. Junior, M. Gmitra, G. M. Sipahi, and J. Fabian, arXiv:1802.06734 (2018).

[60] M. O. Scully and M. S. Zubairy, Quantum Optics (Cambridge University Press, Cambridge, 1997).

[61] H.-P. Breuer and F. Petruccione, The Theory of Open Quantum Systems (Oxford University Press, Oxford, 2002). 